\title{
Notch Signaling Controls Generation of Motor Neurons in the Lesioned Spinal Cord of Adult Zebrafish
}

\author{
Tatyana B. Dias, Yu-Jie Yang (杨宇婕), Kazuhiro Ogai (大貝和裕), Thomas Becker,* and Catherina G. Becker* \\ Centre for Neuroregeneration, School of Biomedical Sciences, University of Edinburgh, Edinburgh EH16 4SB, United Kingdom
}

In mammals, increased Notch signaling is held partly responsible for a lack of neurogenesis after a spinal injury. However, this is difficult to test in an essentially nonregenerating system. We show that in adult zebrafish, which exhibit lesion-induced neurogenesis, e.g., of motor neurons, the Notch pathway is also reactivated. Although apparently compatible with neuronal regeneration in zebrafish, forced activity of the pathway significantly decreased progenitor proliferation and motor neuron generation. Conversely, pharmacological inhibition of the pathway increased proliferation and motor neuron numbers. This demonstrates that Notch is a negative signal for regenerative neurogenesis, and, importantly, that spinal motor neuron regeneration can be augmented in an adult vertebrate by inhibiting Notch signaling.

\section{Introduction}

The spinal cord of adult zebrafish is not a constitutively active neurogenic zone (Reimer et al., 2008), similar to mammals (Yamamoto et al., 2001; Meletis et al., 2008). However, in contrast to mammals, high numbers of neurons are generated from endogenous progenitor cells after a lesion (Reimer et al., 2008). Progenitor cells in the spinal cord of adult mammals are triggered to proliferate by a lesion, but only generate glial cells (Meletis et al., 2008). However, progenitor cells can generate neurons in vitro or when transplanted into neurogenic regions of the CNS (Shihabuddin et al., 2000).

In the mammalian spinal cord, activation of Notch signaling has been proposed to be responsible for the inability of spinal progenitors to generate neurons (Yamamoto et al., 2001). In several developing and adult systems, contact activation of Notch receptors on progenitor cells by Delta or Jagged ligands, present on differentiating progeny or newly recruited progenitors, inhibits proliferation and/or neurogenesis from progenitor cells, thus providing a negative feedback mechanism for their own generation (for review, see Ables et al., 2011; Pierfelice et al., 2011). Indeed, notch 1 expression is increased in the lesioned spinal cord of rats and activation of Notch inhibited, whereas attenuation of

\footnotetext{
Received Dec. 22, 2011; accepted Jan. 17, 2012.

Author contributions: T.B.D., Y.J.Y., T.B., and C.G.B. designed research; T.B.D., Y.-J.Y., K.O., T.B., and C.G.B. performed research; T.B.D., Y.-J.Y., K.O., T.B., and C.G.B. analyzed data; T.B.D., T.B., and C.G.B. wrote the paper.

This work was supported by the Biotechnology and Biological Sciences Research Council (BB/H003304/1), the Robert Packard Center for ALS Research at Johns Hopkins, and the Euan MacDonald Centre for Motor Neurone Disease Research, and TENOVUS Scotland. We thank Dr. Sally Lowell for reagents and critically reading the manuscript, Dr. Pilar Molist for help with the histology, Dr. Trudi Gillespie for expert help with confocal imaging, Drs. Laure Bally-Cuif, Ajay Chitnis, and Julian Lewis for plasmids and transgenic fish, Dr. David Lyons for mutant fish, and Maria Rubio for expert fish care.

*T.B. and C.G.B. contributed equally to the work.

Correspondence should be addressed to either of the following, at the above address: Catherina G. Becker, E-mail: catherina.becker@ed.ac.uk; or Thomas Becker, E-mail: thomas.becker@ed.ac.uk.

K. Ogai's present address: Division of Health Sciences, Graduate School of Medical Science, Kanazawa University, Kanazawa, Ishikawa, 9200942 Japan.

DOI:10.1523/JNEUROSCI.6398-11.2012

Copyright $\odot 2012$ the authors $\quad 0270-6474 / 12 / 323245-08 \$ 15.00 / 0$
}

Notch signaling increased neurogenesis from spinal progenitors in vitro (Yamamoto et al., 2001). Therefore, we asked whether Notch pathway activation also occurs during successful neuronal regeneration in the lesioned spinal cord of adult zebrafish and whether it can be manipulated to generate more motor neurons. This cell type is of major therapeutic interest, because motor neurons are permanently lost after spinal injury and in motor neuron disease in humans.

Following a spinal lesion, $\sim 800$ new HB9-expressing motor neurons are generated from proliferating olig2: $\mathrm{GFP}^{+}$progenitor cells (Reimer et al., 2008). These cells constitute a ventrolateral domain in the ependyma, contact the pial surface with glial endfeet and receive a Sonic Hedgehog signal for motor neuron generation from ventral midline cells (Reimer et al., 2009).

Here we show that increased Notch signaling plays an attenuating role in motor neuron regeneration in the adult zebrafish spinal cord, consistent with a feedback model of Notch activation in progenitor cells. Remarkably, pharmacological inhibition of Notch signaling even increases generation of motor neurons in this adult vertebrate, which may have future therapeutic implications.

\section{Materials and Methods}

Animals. We used wild-type (wik), Tg(olig2:EGFP) (Shin et al., 2003), hereafter designated olig2:GFP, $\operatorname{Tg}(m n x 1: G F P) m l 2$ (Flanagan-Steet et al., 2005), hereafter designated HB9:GFP, $\mathrm{Tg}$ (hsp70l:Gal4), and $\mathrm{Tg}$ (UAS: myc-notch1a-intra) (Scheer et al., 2001) transgenic fish, as well as notchla mutants (notch1 $a^{\text {tp37/tp37) }}$ (van Eeden et al., 1996) of either sex. All experiments were licensed by the British Home Office.

Spinal lesions, adult heat-shocks, and DAPT applications. Complete transection of the spinal cord was performed under visual control as described previously (Reimer et al., 2009). DAPT ( $N$-[N-(3,5-difluorophenacetyl-l-alanyl)]-S-phenylglycine $t$-butyl ester) (Enzo Life Sciences) was injected intraperitoneally, delivering a dose of $50 \mathrm{mg} / \mathrm{kg}$ body weight. Heat-shocks were delivered for $1 \mathrm{~h}$ by slowly $(\sim 2 \mathrm{~h})$ heating the tank water from $26^{\circ} \mathrm{C}$ to $39^{\circ} \mathrm{C}$.

In situ hybridization. Plasmids provided by Julian Lewis (London Research Institute, London, UK), Ajay Chitnis (The Eunice Kennedy 
Shriver National Institute of Child Health and Human Development, Bethseda, MD), and Laure Bally-Cuif (Institute of Neurobiology Alfred Fessard, Gif-sur-Yvette, France) were used to generate probes for notch1a (NM_131441.1), notch1b (NM_131302.2), notch2 (NM_001115094.1), jagged1b(NM_131863.1),jagged2(AF229450.1),deltaA (NM_130954.1), deltaB (NM_130958.1), deltaC (NM_130944.1), deltaD (NM_130955.2), dll-4 (DX504056.1), her1 (NP_571153.1), her4.1 (AAI52181.1), her5(NP_571152.1), her8a (NM_199624), her9 (NP_571948.1), her13 (XM_696748) and hes6 (NM_194400). We used the following primers to generate probes for jaggedla, her4.5 and her12. Jaggedla: Forward primer, 5'-TCG AGA TCG AGG AGT GTG TCG-3', Reverse primer, 5'-GGA GCT GTT AGC ACT GTG TTT GC-3'; Her4.5: Forward primer, 5' -ACA CAA GAC ACA CAG CAA TGG C-3', Reverse primer, 5'-GCC GTG TGG TCA TCG TAT AGA CG-3'; Her12: Forward primer, $5^{\prime}$-CTG TTC GAG CAC AGA CAT GGC-3', Reverse primer, $5^{\prime}$-AAT CCA CCT CCT TCC CAG ACG-3'. All probes showed a characteristic signal in whole-mounted 24-h-old embryos. In situ hybridization on $50 \mu \mathrm{m}$ vibratome sections has been described previously (Reimer et al., 2009).

PCRs. PCRs were performed as described previously (Reimer et al., 2009), using the following primers: Her4.1 Forward primer, 5'-GGC TCA AGA GTT CGT CAA GC-3', Reverse primer, 5' -AGA CGT GTG TGC TTG TCT GC-3'; GAPDH Forward primer, 5' -ACTCCACTCATG GCCGTT-3', Reverse primer, 5' -TCTTCTGTGTGGCGGTGTAG-3'.

Immunohistochemistry and cell quantifications. Anti-HB9 mouse (1: 400, Developmental Studies Hybridoma Bank, University of Iowa, Iowa City, IA), anti-Proliferating Cell Nuclear Antigen (PCNA) mouse (1: 1000, Dako Cytomation), anti-GFP chicken (1:500, Abcam), and antimyc (1:600, Santa Cruz Biotechnology) primary antibodies were used. In some instances, a directly fluorescein-tagged anti-myc antibody (1:1000, Santa Cruz Biotechnology) was used. Secondary antibodies (1:200) were purchased from Jackson Laboratories (Stratech): Cy3 donkey antimouse and Dylight 488 donkey anti-mouse.

Immunohistochemistry on $50 \mu \mathrm{m}$ vibratome sections, with or without prior in situ hybridization, followed a previously established protocol (Reimer et al., 2008). Small (nuclear diameter $<6 \mu \mathrm{m}$ ), intensely labeled $\mathrm{HB}^{+}$cells and ventricular PCNA ${ }^{+}$cells were stereologically counted, as described previously (Reimer et al., 2008). Briefly, confocal image stacks of three randomly selected vibratome sections from the region up to 750 $\mu \mathrm{m}$ rostral to the lesion site and three sections from the region up to 750 $\mu \mathrm{m}$ caudal to the lesion site were analyzed. Cell numbers were then calculated for the entire $1.5 \mathrm{~mm}$ surrounding the lesion site.

For relative values, all Hoechst dye (Hoechst 33342, Biotium)-labeled cells around the ventricle were counted in single optical sections. PCNA and/or myc labeling was determined in the same sections and values were expressed as percentage of all Hoechst-labeled cells. At least 18 optical sections were used from 6 physical sections within $1500 \mu \mathrm{m}$ of the lesion site per fish.

Tests of swimming capability. Endurance of fish was tested by recording the time they were able to hold their position in a water flow $(15 \mathrm{~cm} / \mathrm{s})$, as previously published (Reimer et al., 2009), with the exception that the test duration was limited to $1 \mathrm{~h}$. The spontaneous movements of fish during a 5 min period in standing water were also recorded using an overhead camera and Ethovision (Tracksys) software, as reported previously (Becker et al., 2004).

Statistical analysis. Cell numbers were analyzed using the Mann-Whitney $U$ test if not indicated differently, and behavioral recovery was tested using the two-way ANOVA test, followed by Tukey's post hoc test.

\section{Results}

\section{The Notch pathway is activated in the lesioned spinal cord}

Progenitor cells in the zebrafish spinal cord are located in the ependymal zone around the central canal (Reimer et al., 2008). To determine whether the pathway is active in the lesioned spinal cord, we used in situ hybridization of the hairy-related (her) genes, many of which are direct Notch target genes (Chapouton et al., 2011). Her4. 1 was upregulated at 3 (data not shown) and 14 $\mathrm{dpl}$ (days post-lesion; when motor neuron regeneration peaks; Reimer et al., 2008) from undetectable levels, predominantly in a ventrolateral domain of the ventricular zone. No signal was pres- ent in the ventral midline zone, occupied by sonic hedgehogexpressing ependymo-radial glial cells (Reimer et al., 2009). Her4.5 was upregulated from undetectable levels in scattered cells around the central canal. Her 9 was expressed in dorsal midline cells in the unlesioned spinal cord and this expression domain expanded to comprise the dorsal half of the ventricular zone in the lesioned spinal cord (Fig. 1A,B). Her1, her5, her8a, her12, her13, and hes6 were not detectably expressed in the unlesioned or lesioned spinal cord.

The receptors notch $1 a$ and notch $1 b$ were similarly upregulated from undetectable levels around the central canal, excluding the very dorsal and ventral domains. Notch 2 was upregulated from undetectable levels to a faint signal around the central canal (Fig. 1C).

The notch ligand jagged $1 b$ was upregulated from undetectable levels in ventricular cells of the dorsal midline and the ligand deltaC was upregulated in cells in the vicinity of the ventricular zone, predominantly in the ventral half of the spinal cord (Fig. $1 D)$. None of the Notch ligands deltaA, deltaB, deltaD, dll-4, jagged $1 a$, and jagged 2 were detectably expressed in the unlesioned and lesioned spinal cord. Thus Notch pathway genes are upregulated after spinal lesion in specific dorsoventral domains around the ventricle, probably reflecting the dorsoventral polarity of the adult progenitor domains (Reimer et al., 2009).

Next we tested expression of her4.1 and notch $1 b$ in motor neuron progenitor cells in olig2:GFP transgenic animals. In single optical sections, in situ hybridization signals for both genes coincided with GFP labeling of olig2:GFP ${ }^{+}$ependymo-radial glial cells (Fig. 1E), which are motor neuron progenitor cells (Reimer et al., 2008).

In a HB9:GFP transgenic fish, expression of deltaC mRNA overlapped with small, strongly GFP-expressing, newly generated motor neurons (Reimer et al., 2008) (Fig. 1E). Thus Notch signaling is activated in motor neuron progenitor cells after a spinal lesion, as indicated by upregulation of the target gene her4.1 and the receptor notch $1 b$ in these cells and increased expression of a ligand (deltaC) by the motor neuron progeny.

\section{Notch overactivation inhibits generation of motor neurons and attenuates progenitor cell proliferation in the lesioned spinal cord}

Double-transgenic fish, $T g(h s p 70 l: G a l 4) \times T g(U A S: m y c-n o t c h 1 a-$ intra), in which a heat-shock promoter drives expression of the active intracellular domain of notch $1 a$, fused to a myc epitope, allow conditional and potent overactivation of the pathway (Scheer et al., 2001). Myc immunoreactivity was detected in the spinal cord of unlesioned and lesioned transgenic animals after heat-shocks on 2 consecutive days, but not in heat-shocked wild-type or nonheat-shocked transgenic animals (Fig. 2A). Remarkably, immunoreactivity was predominantly present in ependymo-radial glial cells, as indicated by labeling of radial processes of these cells. This provides some specificity of the manipulation for these spinal progenitor cells. Myc-immunoreactivity was markedly stronger in the nucleus of the cells, indicating correct transport of the activated protein to the nucleus and specificity for ventricular cells was maintained in lesioned animals even after daily heatshocks from 5 to $13 \mathrm{dpl}$ (Fig. 2 B). PCR of spinal tissue surrounding the lesion site indicated increased expression of her4.1 (Fig. 2C). In unlesioned animals, 5 consecutive heat-shocks induced detectability of the target gene her4.1 around the central canal, except for the most ventral cells, by in situ hybridization (Fig. $2 D)$, showing that Notch activation was functional.

To overactivate Notch signaling during motor neuron regeneration, daily heat-shocks were delivered from 5 to $13 \mathrm{dpl}$. This 
A
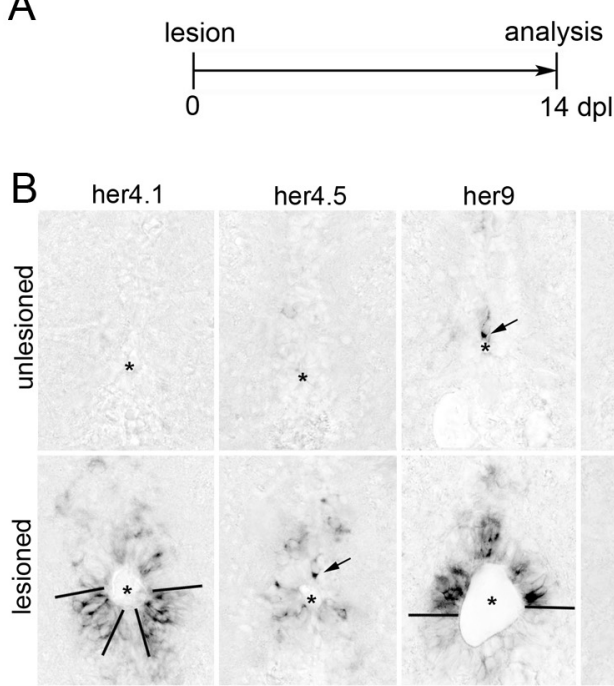

C notch1a

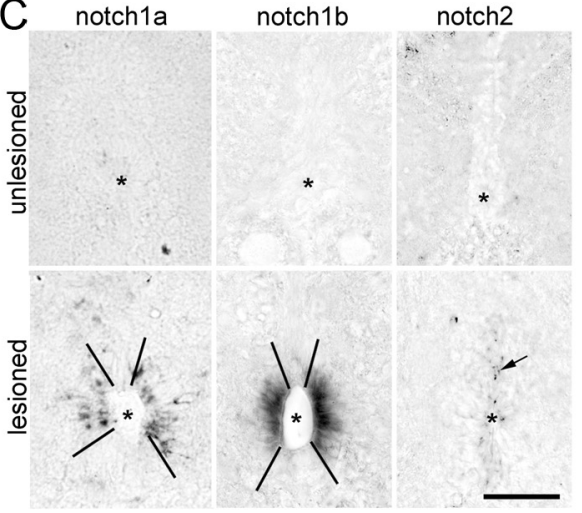

$14 \mathrm{dpl}$
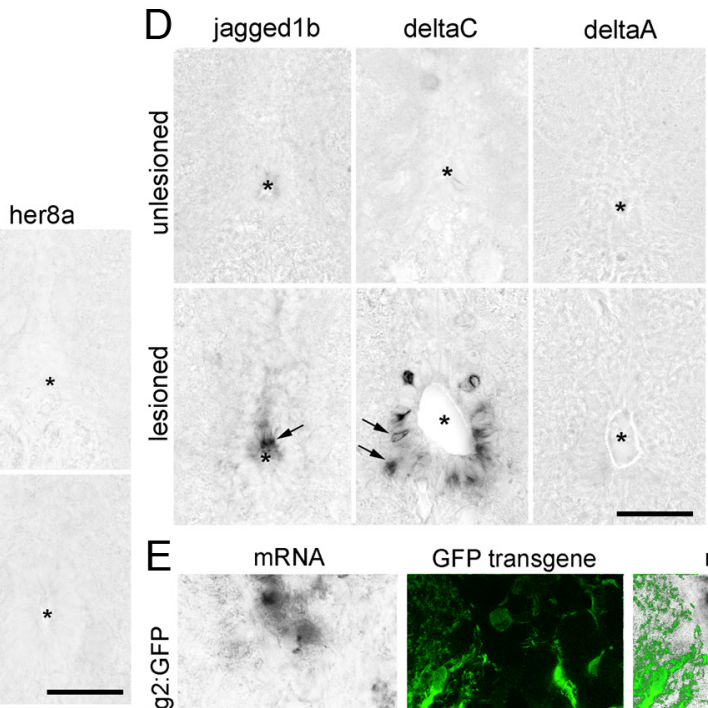

$E$
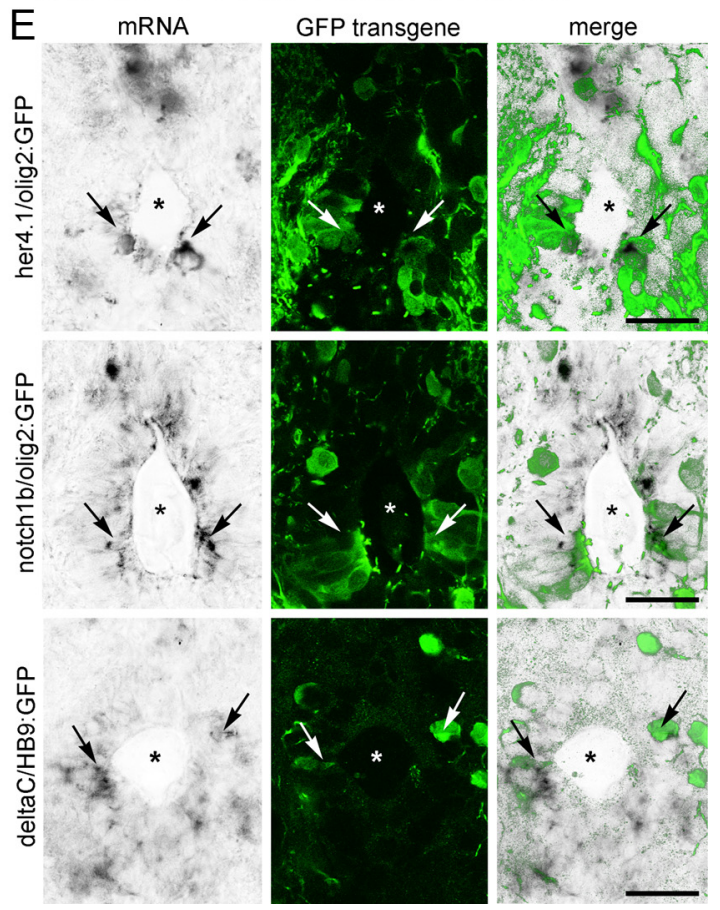

Figure 1. Notch pathway genes are upregulated after spinal injury. In situ hybridizations of spinal cross sections at $14 \mathrm{dpl}$ are shown. Central canal is indicated by asterisks; dorsal is up. In $\boldsymbol{B}-\boldsymbol{D}$, arrows indicate single positive cells and bars delineate expression domains. $\boldsymbol{A}$, Timeline for all experiments. $\boldsymbol{B}$, After a lesion, her4.1, her4.5 and her9, but not her8a, are upregulated in specific areas of the ependymal zone. C, After a lesion, notch $1 a$ and notch $1 b$ are upregulated in specific regions of the ventricular zone. Notch 2 shows weakly increased expression. $\boldsymbol{D}$, After a lesion, $j a g g e d 1 b$ is upregulated only in the dorsal midline, whereas deltaC is upregulated preferentially in cells around the ventral half of the central canal, including cells that are one cell diameter distant from the central canal. DeltaA is not detectably expressed. $\boldsymbol{E}$, Colabeling of transgene and in situ hybridization signal (arrows) indicates her4.1 and notch 16 expression in olig2:GFP ${ }^{+}$ventricular progenitor cells, and deltaC expression in HB9:GFP ${ }^{+}$motor neurons. Scale bars, $25 \mu \mathrm{m}$.

treatment induced detectability of myc-labeling in $29 \%$ of all ventricular cells (Hoechst labeling: $57.61 \pm 2.67$ cells/optical section, myc double-labeled cells: $16.61 \pm 3.53, n=3$ animals) at 14 dpl. The number of small $\mathrm{HB}^{+}$cells in the lesioned spinal cord was determined at $14 \mathrm{dpl}$. Previous BrdU labeling studies have indicated that the vast majority of such cells are newly generated motor neurons, which are rare in the unlesioned spinal cord (Reimer et al., 2008). In lesioned, non-heat-shocked transgenic controls, clusters of intensely labeled small $\mathrm{HB}^{+}$motor neurons were observed in the ventromedial aspect of spinal cross sections (Fig. $3 A, B$ ) in a number that was comparable to previous observations in wild-type animals (Reimer et al., 2008) and not statistically different from that observed in heat-shocked wild-type animals at $14 \mathrm{dpl}$ (data not shown). This supported that the presence of the transgenes or heat-shocks per se did not influence motor neuron generation. However, in heat-shocked transgenic animals, the number of small $\mathrm{HB}^{+}$cells was dramatically re- duced by $87 \%$ in the $1500 \mu \mathrm{m}$ surrounding the lesion site (Fig. $3 B, C)$. Thus, overactivation of Notch signaling strongly inhibits the generation of new motor neurons.

To test whether a lack of motor neuron generation was at least in part due to inhibition of ventricular proliferation we used PCNA immunohistochemistry, which labels cells in early $G_{1}$ phase and $S$ phase of the cell cycle. Proliferating cells were mostly found in the ependymal zone around the central canal, including the motor neuron progenitor domain, as previously reported (Reimer et al., 2008). The overall number of $\mathrm{PCNA}^{+}$cells in the ependymal zone within $1500 \mu \mathrm{m}$ surrounding the lesion site was reduced by $46 \%$ after heat-shocks (Fig. $3 D, E$ ), indicating that Notch activation inhibits progenitor cell proliferation.

At the cellular level we found that in non-heat-shocked double-transgenic animals $25 \%$ of all ventricular cell profiles were PCNA ${ }^{+}$(Hoechst: $61.1 \pm 4.96$ cells/optical section; PCNA double-labeled: $15.22 \pm 4.12, n=4$ ), similar to wild-type con- 
A
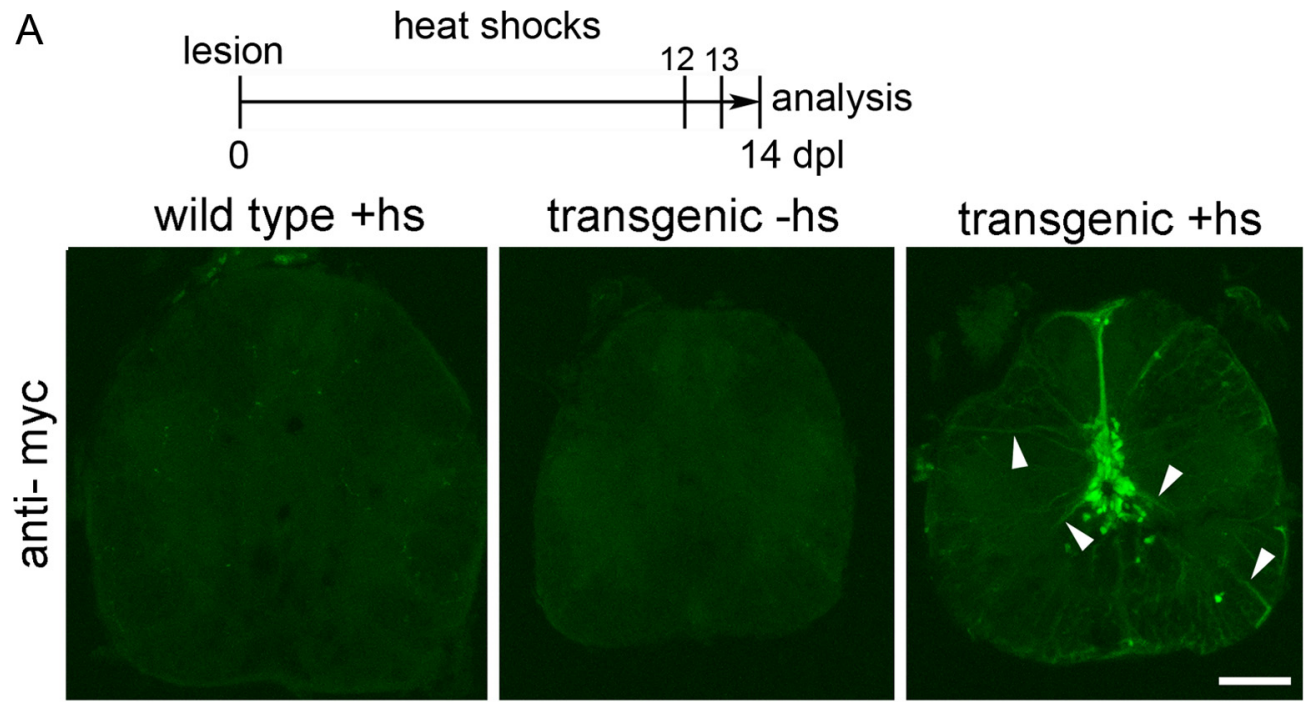

B

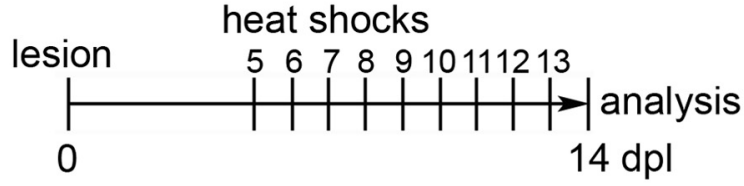

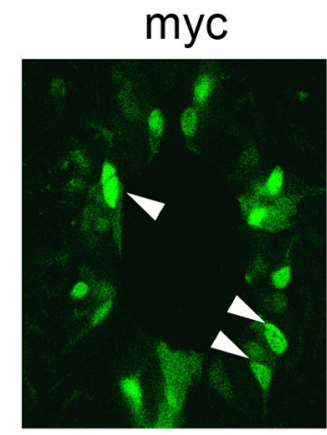

C

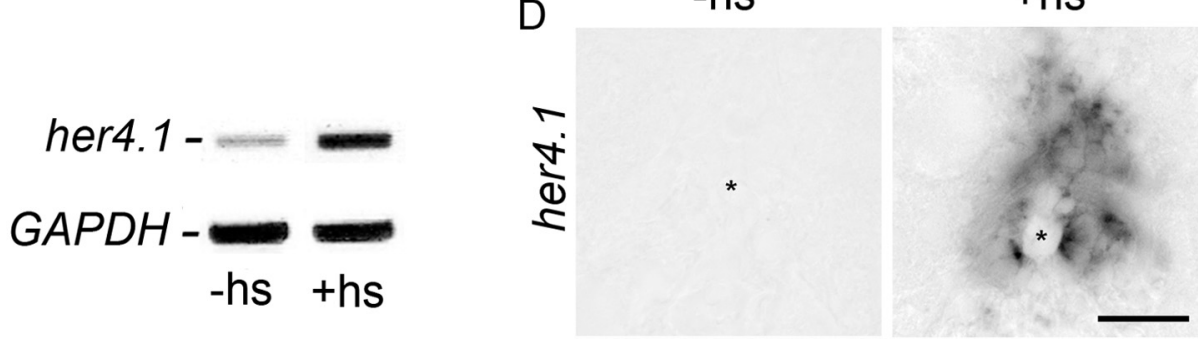

Hoechst

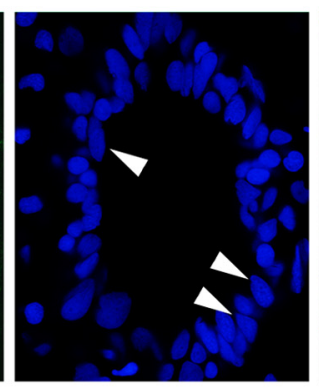

D merge

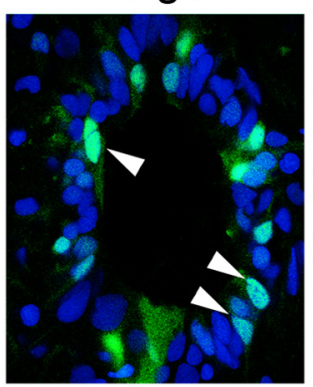

-hs

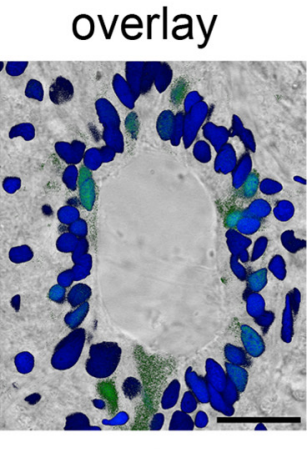

ths

Figure 2. Heat-shock induced overactivation of Notch in ependymo-radial glial cells of $T g(h s p 701: G a l 4) \times T g$ (UAS:myc-notch1a-intra) transgenic fish. Spinal cross sections are shown. $\boldsymbol{A}$, Ventricular myc immunoreactivity is observed in ependymo-radial glial cells and their processes (arrowheads) in double transgenic animals after two heat-shocks (hs). $\boldsymbol{B}$, At higher magnification of a single optical section heat-shocked at the indicated intervals, myc-immunoreactivity is increased in a subset of ependymal cells (see Materials and methods for quantification), mainly in the cells' nuclei (arrowheads). C, Her4.1 expression is increased in the spinal cord of lesioned transgenic animals at $10 \mathrm{dpl}$ after daily heat-shocks from 5 to 9 dpl by PCR. D, In unlesioned animals, 5 daily heat-shocks elicit her4. $1 \mathrm{mPNA}$ expression in the ependymal region, analyzed on day 6. Asterisks indicate the central canal. Scale bars: $\boldsymbol{A}, 50 \mu \mathrm{m} ; \boldsymbol{B}, 25 \mu \mathrm{m} ; \mathbf{D}, 25 \mu \mathrm{m}$.

trols (25\%; Hoechst: $77.52 \pm 9.85$ cells/optical section, $n=3$; PCNA: $19.56 \pm 3.23, n=3$ ) at $14 \mathrm{dpl}$. In heat-shocked doubletransgenic animals, this rate was reduced to $10 \%$ (Hoechst: $57.46 \pm 2.27$ cells/ optical section; PCNA double-labeled cells: $5.85 \pm 1.18 ; n=9$ ), confirming stereological counts. If activation of Notch inhibits cell proliferation, PCNA labeling should be reduced in the same cells that overexpress active Notch. Indeed, only $2.7 \%$ of all conspicuously myc-labeled cells were labeled with PCNA antibodies. This was highly significantly less than the expected value of $25 \%$ (the overall proportion of ventricular cells that are labeled by PCNA antibodies in non-heat-shocked con- trols). The remaining $\mathrm{PCNA}^{+}$cells in heat-shocked animals intercalated with myc-labeled cells (Fig. $3 F$ ). This provides direct evidence that Notch activation in progenitor cells reduces their proliferative activity.

DAPT treatment increases the number of newly generated motor neurons and proliferating progenitor cells in the lesioned spinal cord

The gamma-secretase inhibitor DAPT prevents cleavage of the Notch receptor into its active form and has been shown to attenuate Notch signaling in adult zebrafish (Chapouton et al., 2010). 
A

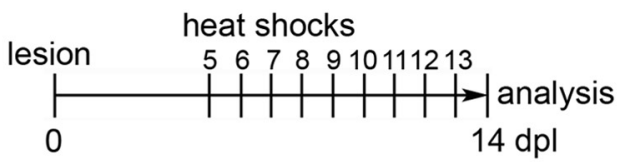

B
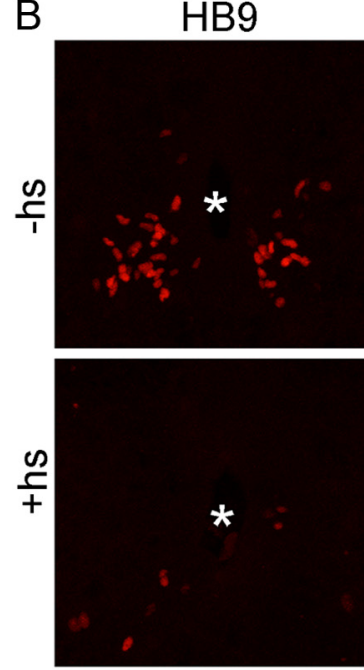

C

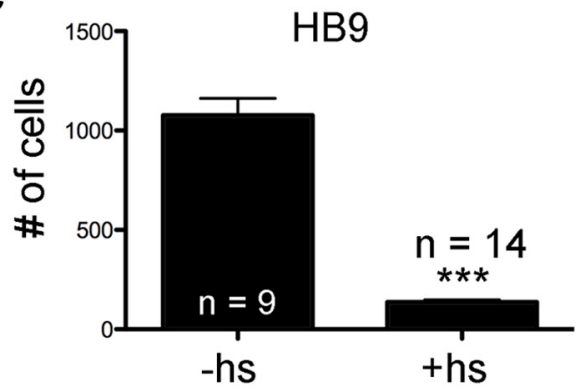

D
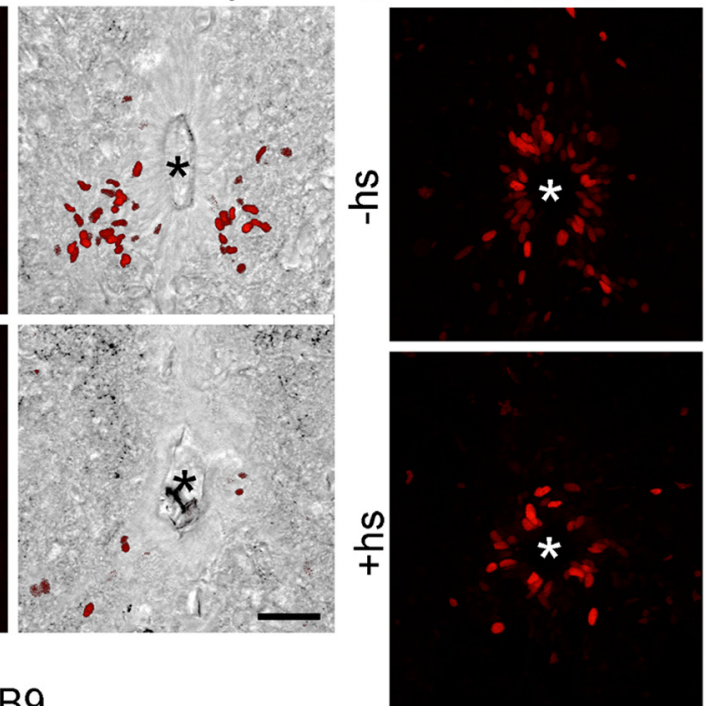

E

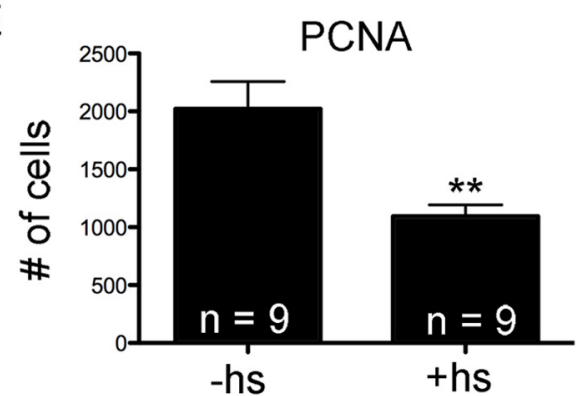

merge

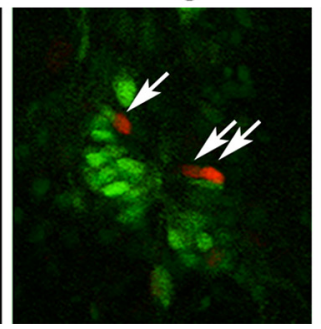

overlay
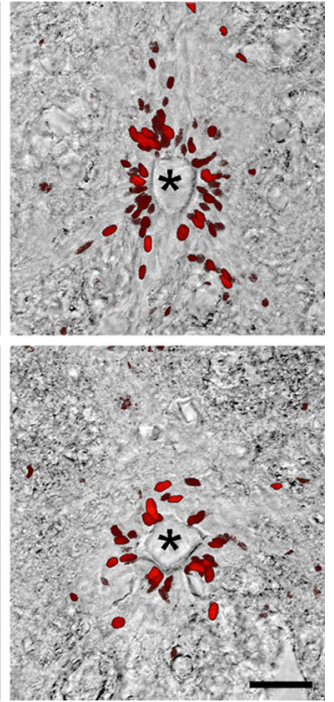

PCNA

Hoechst

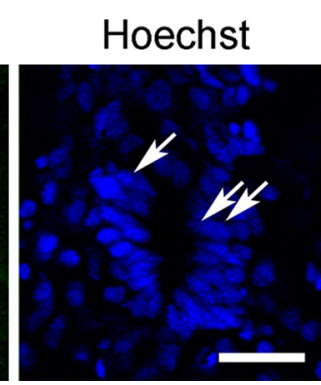

Figure 3. Overactivation of Notch reduces generation of new motor neurons and proliferation in the lesioned spinal cord. Spinal cross sections, centered around the central canal (asterisks in $\boldsymbol{B}$, D), are shown; dorsal is up. $\boldsymbol{A}-\boldsymbol{E}$, The number of small $\mathrm{HB}^{+}{ }^{+}$motor neurons in the ventromedial aspect of the spinal cord $\left(\boldsymbol{B}, \boldsymbol{C}_{;}^{* * *} p<0.001\right)$ and of periventricular $\mathrm{PCNA}{ }^{+}$cells $\left(\boldsymbol{D}, \boldsymbol{E} ;{ }^{* *} p<0.01\right)$ are reduced by the heat-shock (hs) regimen depicted in $\boldsymbol{A}$. $\boldsymbol{F}$, Myc and PCNA (arrows) labeling is mutually exclusive in the ependymal layer. Scale bars, $50 \mu \mathrm{m}$.

Indeed a single intraperitoneal injection at $4 \mathrm{dpl}$ was sufficient to reduce detectability of her4.1 in PCRs of lesioned spinal tissue at $5 \mathrm{dpl}$ (Fig. $4 A$ ), indicating successful reduction of Notch signaling in ventral spinal progenitor cells, in which her4.1 is predominantly expressed.

Injections of DAPT $(3,6,9 \mathrm{dpl})$ during the regeneration period strongly increased the number of newly generated $\mathrm{HB}^{+}$motor neurons by $61 \%$ and that of proliferating $\mathrm{PCNA}^{+}$ventricular cells by $37 \%$ at $14 \mathrm{dpl}$ (Fig. $4 B-F$ ). However, in the adult viable notch $1 a$ mutant, numbers of newly generated motor neurons appeared unaffected at $14 \mathrm{dpl}$ (nonhomozygous siblings: $1244 \pm 114.1$ cells/1500 $\mu \mathrm{m}$ spinal cord, $n=10$ animals; mutants: $1050 \pm 172.5, n=8$ animals, $p>0.05$ ), possibly due to compensation by notch $1 b$. Thus chemically inhibiting all Notch signaling augments progenitor proliferation and motor neuron generation.

As we show here for the first time that the number of newly generated motor neurons can be augmented using DAPT, we wanted to ascertain that the drug had no detrimental effects on subsequent recovery. These could be caused by destroying the ependymal zone as a consequence of progenitor depletion (Carlén et al., 2009) or by off-target toxicity. We used a test in which the swim endurance of fish is measured in a flow of water and assessed the total distance moved within 5 min of spontaneous swimming in an arena (Reimer et al., 2009). DAPT-injected ani- 

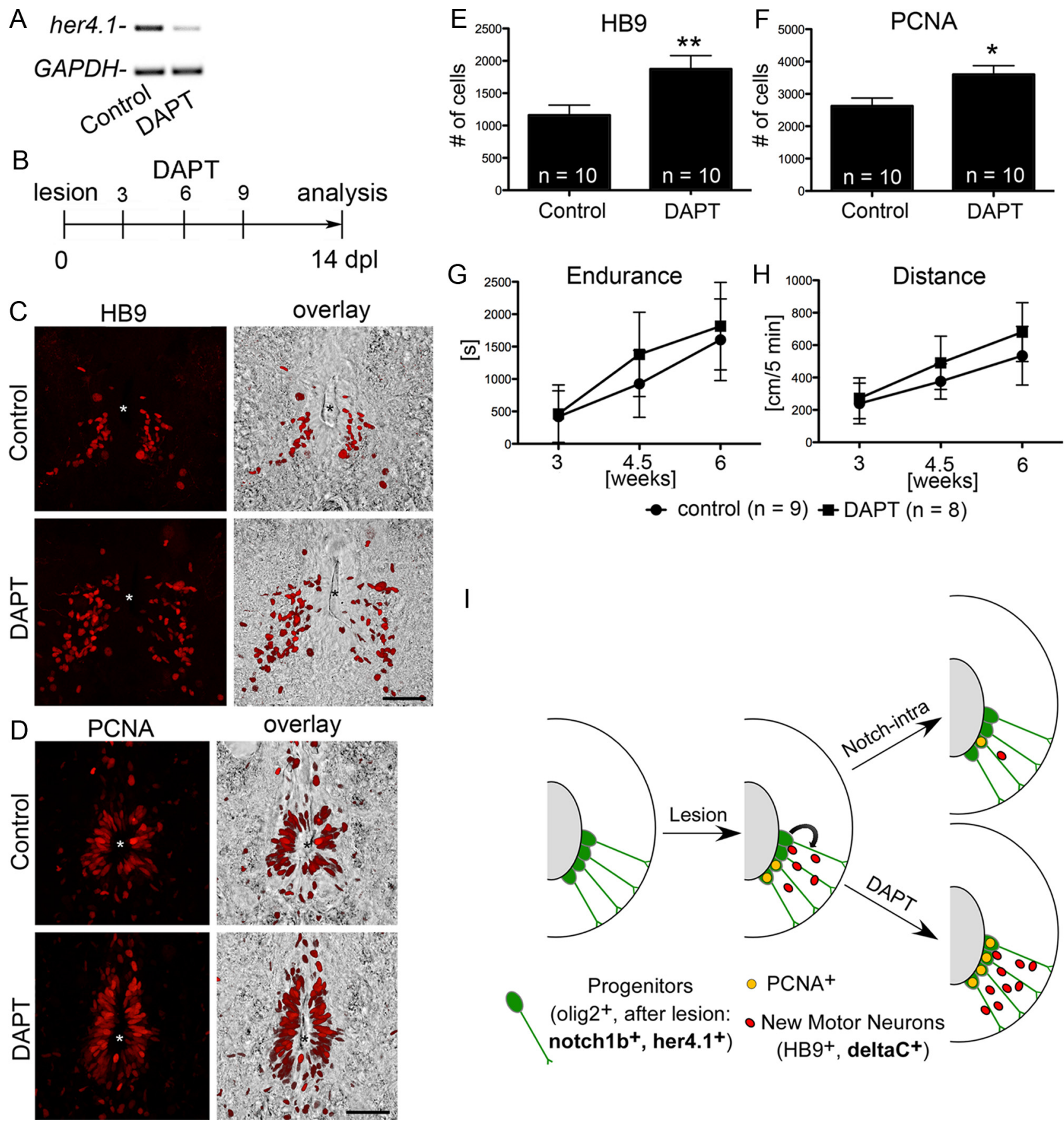

Figure 4. DAPT treatment increases motor neuron generation and ventricular proliferation and does not impair recovery. Spinal cross sections, centered around the central canal (asterisks), are shown; dorsal is up. $\boldsymbol{A}$, A single injection of DAPT at $4 \mathrm{dpl}$ reduces her4. 1 expression at $5 \mathrm{dpl}$ in PCR. $\boldsymbol{B}-\boldsymbol{F}$, The number of small HB9 ${ }^{+}$motor neurons in the ventromedial aspect of the spinal cord $(\boldsymbol{C}$, $\left.\boldsymbol{E}_{;}^{* *} p<0.01\right)$ and of PCNA ${ }^{+}$cells around the central canal $\left(\boldsymbol{D}, \boldsymbol{F} ;{ }^{*} p<0.05\right)$ is increased by the DAPT treatment regimen depicted in $\boldsymbol{B}$. $\mathbf{G}, \boldsymbol{H}$, Endurance in a flow $(\boldsymbol{G} ; \boldsymbol{p}<0.05)$ and the total distance moved $(\boldsymbol{H} ; p<0.01)$ similarly improved over time for DAPT-treated (as in $\boldsymbol{B}$ but with the indicated time points of analysis) and control fish. $\boldsymbol{I}, \mathbf{S c h e m a t i c}$ summary of results. Overexpression of the intracellular domain of Notch1a attenuates, whereas DAPT treatment augments lesion-induced progenitor cell proliferation (PCNA ${ }^{+}$) and generation of motor neurons. Scale bars, $50 \mu m$.

mals significantly recovered in both tests between 3 and 6 weeks post-lesion. DAPT-treated animals appeared to perform slightly better than controls, but this difference was not significant (Fig. $4 G, H)$. This indicates that DAPT has no overt toxic effect on regenerating fish.

Notch manipulations have no detectable effect on progenitor cells in the unlesioned spinal cord

In the unlesioned spinal cord motor neuron generation and ventricular proliferation is rare. Overactivation of Notch by 9 daily heat-shocks (analysis on day 10) did not lead to any changes in the low numbers of small $\mathrm{HB}^{+}$motor neurons (non-heat-shocked: $70.61 \pm 12.11$ cells $/ 1500 \mu \mathrm{m}$ spinal cord, $n=3$; heat-shocked: $50.19 \pm 7.43, n=4 ; p>0.05)$ or $\mathrm{PCNA}^{+}$ cells at the central canal (non-heat-shocked: $64.29 \pm 22.96$ cells/ $1500 \mu \mathrm{m}$ spinal cord, $n=3$; heat-shocked: $65.95 \pm 19.51$, $n=4 ; p>0.05)$. Similarly, daily DAPT injections for 5 consecutive days (analysis on day 8 ) had no influence on the low numbers of small $\mathrm{HB}^{+}$motor neurons (DMSO treated: $137.49 \pm 18.13$ cells $/ 1500 \mu \mathrm{m}$ spinal cord, $n=12$; DAPT 
treated: $156.72 \pm 13.89, n=10 ; p<0.05)$ or $\mathrm{PCNA}^{+}$cells (DMSO treated: $41.35 \pm 5.31$ cells $/ 1500 \mu \mathrm{m}$ spinal cord, $n=$ 12; DAPT treated: $40.31 \pm 5.94, n=10$; $p<0.05)$ at the central canal. This shows that additional signals from the lesion are needed to make spinal progenitor cells competent to react to manipulations of Notch signaling.

\section{Discussion}

We show here that lesion-induced Notch signaling attenuates proliferation of progenitor cells and motor neuron generation from these cells in the lesioned spinal cord of adult zebrafish. We also demonstrate for the first time that motor neuron generation can be augmented in this adult vertebrate by pharmacologically blocking Notch signaling (Fig. 4I, summarized).

Manipulations of Notch activity after a spinal lesion in zebrafish indicate that Notch is a negative regulator of both proliferation in the ventricular progenitor cell zone and of motor neuron generation. This is similar to the role of Notch during constitutive neurogenesis in the forebrain of zebrafish (Chapouton et al., 2010; Rothenaigner et al., 2011) and is compatible with a role of Notch signaling in maintaining progenitor pools. Remarkably, the function of Notch in the regenerating spinal cord of zebrafish is similar to its proposed role in the lesioned spinal cord of rats, where Notch is strongly upregulated and acts as an anti-neurogenic factor in vitro (Yamamoto et al., 2001). It is possible that expression levels of active Notch are too high to allow neurogenesis in the lesioned mammalian spinal cord. As we show here, overactivation of Notch dramatically reduced the number of newly generated motor neurons also in the spinal cord of adult zebrafish. Conversely, in the adult mouse telencephalon, where stroke induces neuroblast formation from ependymal cells, Notch signaling is decreased and ependymal cells are eventually consumed by this differentiation process. Ependymal cells can be rescued by activating Notch (Carlén et al., 2009). However, when we reduced Notch signaling with DAPT, we did not observe any obvious disruption of the spinal ependymal zone and DAPT treatment did not negatively affect recovery of swimming. Such disruption might occur with stronger or longer inhibition of Notch. Overall, our results best support the hypothesis that precise regulation of Notch activity is necessary to allow neurogenesis, while maintaining the progenitor pool in the lesioned CNS.

In the unlesioned spinal cord there was little evidence of neurogenesis (Reimer et al., 2008) and Notch activity. For instance, her4.1 expression, which is positively regulated by Notch signaling (Chapouton et al., 2011), and expression of Notch receptors was undetectable. Similarly, DAPT had no effect on proliferation and motor neuron generation. Even when the active form of Notch was overexpressed, leading to increased her4.1 expression in the ependymal zone, proliferation and motor neuron generation were not influenced. This contrasts with progenitor zones in the adult brain of zebrafish and mice that show constant activity of Notch signaling (Carlén et al., 2009; Chapouton et al., 2010). These observations suggest that ependymo-radial glial cells in the adult zebrafish spinal cord need unknown signals from the lesion event that switch on Notch signaling and neurogenesis (Reimer et al., 2009). This supports an association of Notch signaling with neurogenesis.

Interestingly, the reactivation of Notch signaling is not an exact recapitulation of developmental gene expression. For example, deltaA, deltaD, jagged $1 a$, which are strongly expressed in the embryonic spinal cord, and jagged2, which is expressed in embryonic motor neurons (Yeo and Chitnis, 2007), are not de- tectably expressed in the adult lesioned spinal cord. Vice versa, deltaC is upregulated in the lesioned adult spinal cord, but is hardly detectable in the embryonic spinal cord. Other genes, such as notch $1 a$, notch $1 b$, her4.1 and her4.5 are similarly expressed in development and regeneration (data for embryonic expression not shown and zfin.org).

How does Notch act? We observed that proliferative activity in the ependymal zone was reduced upon overactivation of Notch and increased when Notch signaling was blocked. Thus differences in the numbers of newly generated motor neurons were probably mainly due to differences in proliferative activity of their progenitors. This is similar to constitutively active proliferation zones in the adult zebrafish brain. In that system, a lineage tracing study indicated that manipulations of Notch signaling only influenced the proliferation rate of progenitor cells and not the type of division (symmetrical vs asymmetrical) or cell differentiation (Chapouton et al., 2011). However, we cannot exclude that Notch has additional roles in cell fate decisions of neuroblasts during spinal cord regeneration, similar to development (Shin et al., 2007; Yeo and Chitnis, 2007; Batista et al., 2008; Kimura et al., 2008). In the proliferative zones of the adult mammalian forebrain, Notch signaling either negatively (Imayoshi et al., 2010) or positively (Androutsellis-Theotokis et al., 2006) regulates proliferation, possibly depending on the progenitor cell type analyzed (Pierfelice et al., 2011).

Increased numbers of motor neurons after DAPT treatment did not improve recovery of fish from the spinal lesion. This is perhaps not surprising, as we have shown previously that $<20 \%$ of the newly generated HB9-expressing motor neurons fully differentiate into choline acetyl transferase-positive motor neurons (Reimer et al., 2008) and reducing the number of newly generated motor neurons by $50 \%$ also did not affect recovery (Reimer et al., 2009). Thus the potential beneficial effect of new neurons may be limited by the number of neurons that can be integrated into the system. However, this situation might be different in mammals, in which hardly any neuroblasts are generated in the adult lesioned spinal cord (Shihabuddin et al., 2000; Yamamoto et al., 2001; Meletis et al., 2008).

We conclude that Notch signaling is precisely regulated to allow motor neuron regeneration in the lesioned spinal cord of adult zebrafish. Pharmacological intervention with the Notch inhibitor DAPT increases motor neuron generation from endogenous stem cells, which may inform future therapeutic interventions in the lesioned spinal cord of mammals in which Notch signaling is strong and neurogenesis does not occur (Shihabuddin et al., 2000; Yamamoto et al., 2001; Meletis et al., 2008).

\section{References}

Ables JL, Breunig JJ, Eisch AJ, Rakic P (2011) Not(ch) just development: Notch signalling in the adult brain. Nat Rev Neurosci 12:269-283.

Androutsellis-Theotokis A, Leker RR, Soldner F, Hoeppner DJ, Ravin R, Poser SW, Rueger MA, Bae SK, Kittappa R, McKay RD (2006) Notch signalling regulates stem cell numbers in vitro and in vivo. Nature 442:823-826.

Batista MF, Jacobstein J, Lewis KE (2008) Zebrafish V2 cells develop into excitatory $\mathrm{CiD}$ and Notch signalling dependent inhibitory VeLD interneurons. Dev Biol 322:263-275.

Becker CG, Lieberoth BC, Morellini F, Feldner J, Becker T, Schachner M (2004) L1.1 is involved in spinal cord regeneration in adult zebrafish. J Neurosci 24:7837-7842.

Carlén M, Meletis K, Göritz C, Darsalia V, Evergren E, Tanigaki K, Amendola M, Barnabé-Heider F, Yeung MS, Naldini L, Honjo T, Kokaia Z, Shupliakov O, Cassidy RM, Lindvall O, Frisén J (2009) Forebrain ependymal cells are Notch-dependent and generate neuroblasts and astrocytes after stroke. Nat Neurosci 12:259-267. 
Chapouton P, Skupien P, Hesl B, Coolen M, Moore JC, Madelaine R, Kremmer E, Faus-Kessler T, Blader P, Lawson ND, Bally-Cuif L (2010) Notch activity levels control the balance between quiescence and recruitment of adult neural stem cells. J Neurosci 30:7961-7974.

Chapouton P, Webb KJ, Stigloher C, Alunni A, Adolf B, Hesl B, Topp S, Kremmer E, Bally-Cuif L (2011) Expression of hairy/enhancer of split genes in neural progenitors and neurogenesis domains of the adult zebrafish brain. J Comp Neurol 519:1748-1769.

Flanagan-Steet H, Fox MA, Meyer D, Sanes JR (2005) Neuromuscular synapses can form in vivo by incorporation of initially aneural postsynaptic specializations. Development 132:4471-4481.

Imayoshi I, Sakamoto M, Yamaguchi M, Mori K, Kageyama R (2010) Essential roles of Notch signaling in maintenance of neural stem cells in developing and adult brains. J Neurosci 30:3489-3498.

Kimura Y, Satou C, Higashijima S (2008) V2a and V2b neurons are generated by the final divisions of pair-producing progenitors in the zebrafish spinal cord. Development 135:3001-3005.

Meletis K, Barnabé-Heider F, Carlén M, Evergren E, Tomilin N, Shupliakov O, Frisén J (2008) Spinal cord injury reveals multilineage differentiation of ependymal cells. PLoS Biol 6:e182.

Pierfelice T, Alberi L, Gaiano N (2011) Notch in the vertebrate nervous system: an old dog with new tricks. Neuron 69:840-855.

Reimer MM, Sörensen I, Kuscha V, Frank RE, Liu C, Becker CG, Becker T (2008) Motor neuron regeneration in adult zebrafish. J Neurosci 28:8510-8516.

Reimer MM, Kuscha V, Wyatt C, Sörensen I, Frank RE, Knüwer M, Becker T, Becker CG (2009) Sonic hedgehog is a polarized signal for motor neuron regeneration in adult zebrafish. J Neurosci 29:15073-15082.

Rothenaigner I, Krecsmarik M, Hayes JA, Bahn B, Lepier A, Fortin G, Götz M, Jagasia
R, Bally-Cuif L (2011) Clonal analysis by distinct viral vectors identifies bona fide neural stem cells in the adult zebrafish telencephalon and characterizes their division properties and fate. Development 138:1459-1469.

Scheer N, Groth A, Hans S, Campos-Ortega JA (2001) An instructive function for Notch in promoting gliogenesis in the zebrafish retina. Development 128:1099-1107.

Shihabuddin LS, Horner PJ, Ray J, Gage FH (2000) Adult spinal cord stem cells generate neurons after transplantation in the adult dentate gyrus. J Neurosci 20:8727-8735.

Shin J, Park HC, Topczewska JM, Mawdsley DJ, Appel B (2003) Neural cell fate analysis in zebrafish using olig2 BAC transgenics. Methods Cell Sci 25:7-14.

Shin J, Poling J, Park HC, Appel B (2007) Notch signaling regulates neural precursor allocation and binary neuronal fate decisions in zebrafish. Development 134:1911-1920.

van Eeden FJ, Granato M, Schach U, Brand M, Furutani-Seiki M, Haffter P, Hammerschmidt M, Heisenberg CP, Jiang YJ, Kane DA, Kelsh RN, Mullins MC, Odenthal J, Warga RM, Allende ML, Weinberg ES, NüssleinVolhard C (1996) Mutations affecting somite formation and patterning in the zebrafish, Danio rerio. Development 123:153-164.

Yamamoto S, Nagao M, Sugimori M, Kosako H, Nakatomi H, Yamamoto N, Takebayashi H, Nabeshima Y, Kitamura T, Weinmaster G, Nakamura K, Nakafuku M (2001) Transcription factor expression and Notchdependent regulation of neural progenitors in the adult rat spinal cord. J Neurosci 21:9814-9823.

Yeo SY, Chitnis AB (2007) Jagged-mediated Notch signaling maintains proliferating neural progenitors and regulates cell diversity in the ventral spinal cord. Proc Natl Acad Sci U S A 104:5913-5918. 